\title{
Vídeo digital : o estado da arte e possibilidades no ensino brasileiro
}

\section{Solange Puntel Mostafa} Rosa Maria Vivona Bertolini

\section{Resumo}

Este artigo apresenta o projeto da PUCCampinas proposto à Capes, através do seu programa de integração entre pósgraduação e graduação o desenvolvimento de uma videoteca digital. $\mathrm{O}$ projeto nasceu inspirado nas videotecas que começam a aparecer no Brasil como as relatadas no projeto original. Além disso, enfoca discursos na literatura, extraídos de artigos técnicocientíficos do projeto Informedia, relatado por professores que estão construindo videotecas para suas disciplinas nos Estados Unidos.

\section{Palavras-chave}

Videoteca digital; ProfessoresTecnologia; Projeto Informedia

\begin{abstract}
This article presents the project of PUCCampinas proposed at Capes, through its integration program between masters degree and undergraduate program in the development of a digital videoteca. The project was born inspired by the video libraries that begin to appear in Brazil and in the american experimental digital video libraries project called "Informedia". The authors describe these experiences and its utilization for higher education at local context.
\end{abstract}

\section{Keywords}

Digital videoteca; Teachers-Technology; Informedia Project 


\section{INTRODUÇÃO}

As inovações tecnológicas na área de vídeo digital estão sendo desenvolvidas nos Estados Unidos, no interior de um projeto chamado "Informedia Digital Video Library" 11 Tal projeto está baseado

em vasto programa de pesquisa que congrega engenheiros, tecnólogos, cientistas da computação, psicólogos, sociólogos, bibliotecários (em menor quantidade) e comunicólogos.

O tema das videotecas tem muito a ver com as Bibliotecas Digitais e dentro do programa americano esses projetos estão tendo um significado bastante específico: buscam refletir o mundo real, apoiando-se em testbeds, que são sistemas prototipados, com coleções reais de documentos e grupos reais de usuários, ambos em quantidades representativas, suportadas por financiamento de pesquisa.

Os objetivos das atividades em Testbeds e aplicações focalizam o desenvolvimento de protótipos para teste, demonstração e validação de tecnologias e, como recursos para comunidades especializadas, tanto técnicas como não técnicas. Os projetos de aplicações devem resultar em ambientes duradouros de pesquisa e aprendizagem sobre o uso público, de forma criativa, das bibliotecas digitais.

${ }^{1}$ THE INFORMEDIA DIGITAL VIDEO LIBRARY: NSF PROPOSAL: PROJECT DESCRIPTION.

Disponibilizado em:

http://www.informedia.cs.cmu.edu/info/imsummary.html

DIGITAL LIBRARIES INITIATIVE. Available

research. Disponibilizado em:

http://dli.grainger.uiuc.edu/national.htm
As revistas por onde está fluindo a vasta produção científica do grupo também abrangem áreas diversas; os artigos estão aparecendo em revistas do tipo Computer , Multimedia Systems, Computer Vision and Pattern Recognitionm, Journal of Visual Communication and Image Representation, Mutimedia Tools and Aplications, D-Lib Magazine, ACM Computer Surveys e as revistas de engenharia, as IEEE de todo tipo.

Um ou outro texto aparece em revistas de Ciência da Informação, a exemplo do recente texto de WITBROCK e HAUPTMANN (1998) publicado no JASIS (Journal of the American Society for Information Science). $\mathrm{O}$ título do artigo justifica o seu deslocamento em relação à Ciência da Informação e Biblioteconomia ("Speech recognition in a Digital Video Library").

É de se notar, no entanto, que quando ainda nem se falava em Bibliotecas Digitais, já aparecia no Journal of Documentation, uma revista tão cara aos cientistas da informação europeus, artigos como os de O'CONNOR falando em "concepts and proposals for surrogates for films" (1985).

O projeto Informedia é pioneiro na criação de videotecas digitais. Desenvolveu-se a integração entre reconhecimento de voz, processamento de imagens e processamento de texto para automaticamente representar os conteúdos dos vídeos.

Da mesma forma que o texto precisa ser representado por palavras-chaves ou resumos, os vídeos precisam ser representados por imagens-chaves ou "resumos" os quais tiveram a denominação dominante, no interior da produção científica do projeto, de 
"multimedia abstractions" (CHRISTEL et al., 1998 ; CHRISTEL, 1997a, 1997b); outros autores, porém usaram a mesma terminologia textual, "surrogates" (KOMLODI e SALUGHTER, 1998) e outros ainda "video abstracts" (LIENHART et al., 1997).

Qualquer das terminologias estava referindo-se a um conjunto de técnicas de representação de imagem e som. E se falamos em representação ou resumo estamos falando, no complexo caso das imagens sonoras em movimento, em processos de compressão e em formas variadas de representação e alinhamento entre imagem, som e velocidade.

Assim, as pesquisas frutificaram em áreas como o processamento de imagens (onde várias técnicas estão sendo descritas na literatura), o processamento de som com o reconhecimento de fala (WITBROCK, 1998a,1998b), a segmentação e alinhamento do diálogo falado com os diálogos transcritos (HAUPTMANN, 1998) e a detecção do silêncio para melhor realizar o "video skim", expressão cunhada por CHRISTEL et al. (1998) que assim a define:

\begin{abstract}
Nós definimos "video skim" como uma abstração temporal $e$ multimidia que incorpora ambas as informações de video e audio de uma extensa fonte. Um "video skim" é tocado (played) mais do que visto estaticamente e 2 minutos de "skim" pode representar 20 minutos do original.
\end{abstract}

Na classificação posterior de CHRISTEL et al. (1999) as "multimedia abstractions" se dividiriam em três tipos: a) imagens individuais representativas do conteúdo do vídeo com pequenos títulos escritos; nota-se que essas imagens são selecionadas automaticamente através de várias técnicas; b) imagens seqüenciais e dispostas como num rolo de filme "filmstrips" e as "abstrações" do tipo "video skims ... which are played so that over time video and audio information from a longer source is presented" (CHRISTEL et al., 1999: p.19)

Com relação ao processamento de imagem, vários detalhamentos tem sido tentados: ROWLEY (1995) e HOUGHTON (1999) por exemplo "experimentam" a identificação de faces (rostos) enquanto GONG (1998) tenta a recuperação de imagens por imagens baseada em texturas e cores.

Há exemplos de pesquisa relatando apenas a técnica de selecionar os quadroschaves (key frame) para posterior brauseio e a compreensão do usuário com relação à apresentação dos quadros (se fixos, se em movimento) KOMBOLI, MARCHIONINI (1998) e DING (1997).

Algumas pesquisas parecem confirmar o senso comum, por exemplo DING (1997) ao testar a velocidade na apresentação das imagens ao usuário, verifica que imagem fixa é melhor na tarefa de identificação de um objeto enquanto que imagem em movimento dá melhor compreensão do contexto (gist comprehension).

Essas pesquisas só podem ser realizadas após a videoteca estar disponibilizada em vários formatos de saída, a exemplo do CHRISTEL (1996) no texto Informedia goes to school.

São coleções "prototipadas" como explicado na primeira parte desta revisão. E por isso subentitulamos o projeto das Bibliotecas Digitais como um todo como "um projeto peculiar de pesquisa". As 
pesquisas realizam-se após toda a experimentação da inovação tecnológica, que de per si, envolve também muita experimentação científica no lado das tecnologias.

Estratégias de como dispor os resultados de uma busca é um dos aspectos mais importantes dos grandes sistemas de recuperação da informação. Uma estratégia ideal otimiza um processo de filtragem, equilibra a quantidade de informação e ajuda o usuário a ter resultados mais precisos com o menor esforço.

O "brauseio" através de substitutos adequados possibilita aos usuários fazerem um julgamento preliminar de relevância entre um grupo de documentos recuperados e a tempo de decidir se querem examinar mais atentamente os objetos informacionais.

Os vídeos, considerados como imagens em movimento, vídeos e videoclips precisam ser acessíveis através de um mecanismo de busca e de browsing. Considerando as distinções entre sistemas de representação por palavras e por imagens, os substitutos e os mecanismos de controle tradicionais não são suficientes para os usuários no processo de selecionar vídeos.

O'CONNOR (1991) propôs os quadroschaves (video key frames) para funcionar como os "resumos" dos vídeos. Os quadros-chaves são figuras fixas representativas de diferentes cenas contidas em cada sequiência de vídeo, baseada nas propriedades físicas $\mathrm{e}$ semânticas do vídeo. Os quadros são vantajosos em relação aos substitutos de documentos textuais.
Como apontado por O'CONNOR em pesquisas seguidas (1985, 1986a, 1986b) são formas sistemáticas de traduzir imagens em palavras. Palavras são signos gerais, conectadas por convenção a um conceito, chegando-se a mais especificidade pela sintaxe. Enquanto as figuras ou quadros são específicos e chega-se a mais especificidade pelo seu contexto, que age para reduzir atributos únicos de um objeto a atributos gerais de uma classe de objetos.

Vídeos integram informação gráfica, dinâmica e verbal ambas espacialmente e temporalmente. DING (1997) investiga os parâmetros para projetar interfaces nas bases de dados de vídeo que permitam aos usuários "brausear" esses substitutos.

Usando quadros chaves ("key frames") como substituto do documento e baseado no desempenho do usuário, DING (1997) examina qual a velocidade ótima de apresentação de quadros fixos ou em movimento em duas situações cognitivas já categorizadas e freqüentes nessas pesquisas de vídeo: identificação de um objeto (Object Identification) e compreensão contextual da cena (Gist Comprehension)

Além disso, o vídeo provavelmente contem uma abundância de informações convertidas ambas em sinal de vídeo (movimento de câmara, mudança de cena, cores) e sinais de áudio (barulho, silêncio, diálogo) (CHRISTEL 1995). Desconsiderando que a ambigüidade da linguagem natural pode trazer viés na interpretação do vídeo, muitas informações no vídeo não podem ser descritas apropriadamente por palavras.

A segmentação de vídeo e as técnicas de extração dos quadros-chaves contam com $90 \%$ de acerto na avaliação de 
ENGLANDE et al. (1996). Por isso essas técnicas tem sido aplicadas em vários projetos de indexação e recuperação de video (KOBLA et al., 1996; TEODOSIO e BENDER, 1993) .

Quadros-chaves com suas relações caracteriza a estrutura do vídeo. Além disso, o volume de quadros-chave é muito menor que um vídeo clip em formato digital. Quadros-chaves podem ser dispostos estaticamente ou dinamicamente. Quadros dinâmicos referem-se a rápido "playback" mas os objetos podem ser vídeo clips inteiros, vídeos skims ou quadros-chaves do vídeo.

São pois desenvolvimentos bastante avançados que estão sendo conseguidos no escopo do projeto Informedia das Videotecas Digitais, desenvolvimentos em sua maioria tecnológicos, o que dificulta nossa compreensão mas não dispensa a re (visão). Até para situarmonos em relação ao Proin-2.

\section{ÁREAS DE PESQUISA DAQUI PRA FRENTE}

Toda a base tecnológica desenvolvida até aqui aponta três grandes áreas de pesquisa na sequiência e na consequiência do realizado até aqui. Vários pesquisadores em várias universidades americanas estarão desenvolvendo o "realizado até aqui" ainda pelas próximas décadas) . Mas três áreas novas se apresentam, como demonstrado por WACTKAR, 1999:

- desenvolvimento de videotecas multilinguais com busca em várias línguas;

- indexação numa área que é desestruturada e não editada ainda ;

- a integração de videotecas nos processos de auto-sumarização e visualização (o mesmo sonho das bibliotecas textuais) .

\section{OS DESAFIOS PARA O SÉCULO 21}

O grande desafio será trabalhar com dados e informações sem ter que entrar em considerações sobre sua localização física na rede ou sobre peculiaridades notacionais e sintáticas. Será o século que desenvolverá ferramentas que permitam operar com conceitos sobre coleções de dados parcialmente armazenados em milhões de repositórios distribuídos pelo mundo todo.

Isso vai requerer a existência de índices semânticos atribuídos a cada repositório e de ferramentas auxiliares que permitam operar esses índices de tal modo que usuários consigam chegar à informação desejada não somente quanto ao assunto escolhido mas também quanto ao grau de detalhamento e tipo de uso pretendido para a informação.

Quando isso acontecer a Internet terá se transformado no Interspace, onde usuários navegam por espaços abstratos correlacionando diferentes fontes para encontrar a informaçãa mais apropriada para suas necessidades

Se entendemos bem a literatura dos países e autores avançados tecnologicamente, a idéia não é substituir livros e revistas tradicionais, mas sim oferecer recursos que possam suplementá-los, principalmente no que diz respeito a informações de natureza não textual (tais como mapas e fotos, no caso do Geographical Information System - GIS da Universidade da Califórnia) e

\footnotetext{
${ }^{2}$ Scgatzm Bruce \& Chen, Hsinchun. IEEE Computer, 1999 fev. p.45-50.
} 
dinâmica (tais como voz, música e imagens em movimento) como na Mellon University.

Ao tornar o conteúdo disponível em meio eletrônico, as bibliotecas digitais agregam valor e conteúdo à infra-estrutura da Internet. A biblioteca digital também deve ser tão fácil de acessar quanto a biblioteca pública tradicional, para que o cidadão comum, em qualquer etapa de seu desenvolvimento pessoal ou profissional se beneficie do seu conteúdo.

\section{O PROIN-98 DA PUC-CAMPINAS}

A PUC-Campinas propôs à Capes através do seu programa de integração entre pósgraduação e graduação o desenvolvimento de uma videoteca digital. O projeto nasceu inspirado nas videotecas que começam a aparecer no Brasil como as relatadas no projeto original. Especialmente a videoteca de artes da UNICAMP, cuja organizadora enfatiza a estrutura de banco de dados utilizada, que segunda a autora é inédita entre as congêneres (FAGUNDES, 1999).

Inspirou-nos também o forte apelo da Educação à Distância especialmente dentro da política educacional da PUCCampinas; vimos que nos cursos de EAD, as apresentações em vídeo (resumidas em "multimidea abstractions") podem ser valiosas contribuições em clips rápidos para várias áreas de conhecimento e em várias situações.

Posteriormente, verificamos que professores americanos de várias áreas reuniam-se em grupos de discussão para compartilhar como os video clips poderiam ser acrescentados aos seus sites de cursos na Web (http://leahi.kcc.hawaii.edu/org/wwwdev/ logs/2919.html). Diálogos como os que se seguem encontram-se nos arquivos das grupos 3

"I am considering adding some lecture clips to my course web site and would appreciate any input you might have as to what format I should be looking at - real video, - avi's, -mov's, -mpg's ?"

\section{Resposta:}

"For the web: mov's for Quick Time streams on the web well, but we think it might be limited to 5 minutes or less. Example:

http://www.edb.utexas.edu/tp99/testing/mo dule/module.10.html"

"rm's for Real Audio streaming video for longer segments. (requires a Real Audio Server, so without access to that go with the Quick Time) Example: http://www.edb.utexas.edu/tp99/testing module2/module2.2.thml

Há também na literatura, além de diálogos como os demonstrados acima e além dos artigos técnico-científicos do projeto Informedia, há relatos de professores que estão construindo videotecas para suas disciplinas. Por exemplo, o professor de educação física coleta imagens de acidentes musculares para montar a videoteca digital. "What is unique about this library is that the video is of unburies as they are occurring" http://www.uwec.edu/academic/cps/hum anity3/3-04.html 


\section{SOBRE FORMATOS E} LINGUAGENS

As preocupações dos teóricos de Bibliotecas Digitais recaem, como observou bem FAGUNDES (1999: p.34) em sete itens: arquitetura técnica, construção de uma coleção digital, digitalização, metadado ou metadocumentos, nome endereço, direitos autorais e preservação.

Á digitalização compete definir os formatos e linguagens que viabilizam a codificação dos textos, sons e imagens. Digitalização é um termo recente que "significa converter para o formato eletrônico um dado que esteja armazenado em sistema analógico ou um suporte fixo (livro, jornal, foto, pintura, filme, vídeo, áudio). Significa também selecionar formatos para arquivar dados $e$ os disponibilizar em rede..." (FAGUNDES, 1999: p.36)

Textos por exemplo são codificados em linguagens como a HTML (Hypertext Markup Language); Imagens e sons podem ser armazenados e comprimidos em diferentes formatos (Mpge-1, QuickTime e RealVideo) e envolve um volume muito grande de dados donde vem a necessidade de comprimir os arquivos . "Por esta razão no momento da captura digital de um vídeo ou de uma fonte sonora, já devem ser estabelecidos os parâmetros de compressão." (FAGUNDES, 1999: p.39).

Por causa desta especificidade das imagens de lotarem a capacidade de armazenamento dos computadores, o desenvolvimento das tecnologias de imagens ainda encontram-se em fase de testes, como evidenciam as várias pesquisas do projeto Informedia.
Dentre as tecnologias já desenvolvidas, a tecnologia do RealVideo "possibilita ao usuário ver e ouvir o arquivo vídeo ou áudio (através do RealAudio) no momento em que suficiente conteúdo (o que demora apenas alguns segundos) tenha chegado, não necessitando ser carregado por completo. Essa solução é chamada de streaming media." (FAGUNDES, 1999 : p.39)

Esta tecnologia veio substituir o Mpge e o Quick Time mas com perda de qualidade e informação. "Ao clicar em um item de streaming media, o download do arquivo começa. Mas, ao invés de ter que carregar o arquivo inteiro no computador para que ele possa ser visto (ou ouvido), ele pode começar a ser rodado no momento em que suficiente conteúdo (comumente chamado buffer) tenha chegado e assegurado um braoadcasting contínuo, e isto demora apenas alguns segundos." (FAGUNDES, 1999 : p.49)

\section{APRESENTANDO A VIDEOTECA DIGITAL}

No ambiente da PUC-Campinas deverá ser utilizada a técnica de keyframe ou quadros-chaves em movimento para a apresentação primária do conteúdo dos vídeos que por sua vez poderão ser consultados na página principal de acesso á videoteca, através de relação alfabética simples de títulos. A partir desta listagem, será acrescida então, link para a imagem primária dos quadros-chaves.

As questões ergonômicas de construção do site da videoteca serão tratadas por especialistas do NAR-Núcleo de Assessoramento Remoto da PUCCampinas. Detalhes como frames, barras de rolagem e bottons deverão ser utilizados com reserva para evitar 
poluição visual. Destaque deverá ser o quadro-chave, que por si só, preencherá a área de tela. Preferencialmente, os vídeos digitais deverão ser assistidos em monitores de tela plana e de dimensão superior a 15 polegadas. A compreensão contextual da cena e o tempo de exibição que inicialmente será de 2 minutos, serão definidos após testes com diferentes tipos de usuários, observando pesquisa de DING (1998).

Neste processo deverá ser delineado um cenário com a atuação de diferentes atores:- informáticos, educadores e bibliotecários para que as diferenciadas percepções dos usuários possam ser compreendidas e retratadas em tempo ótimo de exibição de quadros-chaves permitindo que a apreensão de conteúdos seja feita no menor tempo possível.

Quanto ao acesso, será habilitado de duas formas distintas: Intranet e Internet. Em termos de Intranet, o usuário, ao entrar no site da videoteca, fornecerá sua senha de acesso (registro acadêmico ou funcional) habilitando-o a navegar por todas as páginas e opções disponíveis na homepage. Em Intranet, a permissão de acesso é para a visualização integral do vídeo, bastando para isso clicar no título escolhido sendo remetido, através de link para o quadro-chave e através de duplo clique será iniciado o processo de download do arquivo, sendo utilizado o recurso de streaming media que permitirá a visualização em poucos segundos, como citado anteriormente.

Pretende-se ainda, complementar o serviço de Videoteca com uma linha de pesquisa em Indexação de imagens, criando grupos de controle que serão expostos a duas diferentes técnicas de recuperação : quadros-chaves e resumos convencionais.
A técnica de quadros-chaves procura traduzir imagens em palavras enquanto os resumos são oriundos de análise de palavras e compreensão de conteúdo retiradas de texto escrito, no nosso caso, da própria sinopse.

A pesquisa deverá nortear o futuro da videoteca digital em termos de indexação e recuperação de imagens.

Em se tratando de acesso extra-muros, o usuário, pela Internet, estará habilitado a visualização parcial, ou seja, ao selecionar o vídeo através de listagem que o remeterá aos quadros-chaves poderá assistir apenas aos 2 minutos de imagens selecionadas. Futuramente, pretende-se disponibilizar o acesso integral dos vídeos para a comunidade externa, conquanto que os resultados do projetopiloto e da pesquisa sobre indexação e recuperação tenham sido altamente satisfatórios já que a tecnologia de streaming permite rapidez no acesso ao vídeo, dependendo então, apenas dos recursos de hardware e software utilizados pelos usuários.

\section{PARA QUE A VIDEOTECA DIGITAL ?}

Numa retrospectiva na área de tecnologia educacional, observar-se-á que a incorporação de novos elementos didáticos-pedagógicos ao modo de ensinar tem-se dado com uma lentidão que não agrada nem aos educadores nem tampouco aos educandos. Considerando que, de um modo geral as novas tecnologias demoravam de 30 a 50 anos para se configurar em uso, passando para tempo de dez anos e após o surgimento da Internet este intervalo está alcançando a marca de 30 dias entre o surgimento e a incorporação de novas tecnologias não cabe discussão se este novo recurso será ou não utilizado pelos educadores como 
elemento pedagógico. Consolidado está o uso de vídeos em sala de aula e ambientes educacionais, de treinamento, de lazer etc. Trata-se apenas de uma nova roupagem com grandes vantagens para os usuários. É uma nova maneira de olhar e usar o velho.

\section{REFERÊNCIAS BIBLIOGRÁFICAS ${ }^{4}$}

CHRISTEL, M. et al. Evolving video skims into useful multimedia abstractions. In: CHI'98 CONFERENCE ON HUMAN FACTORS IN COMPUTING SYSTEM, 1998, Los Angels. Proceedings..., Los Angeles : [s.n.], 1998. p 171-178.

CHRISTEL, M., HAUPTMANN, A., WARMACK, A., CROSBY, S. Adjustable filmstrips and skims as abstractions for a digital video library. In: IEEE ADVANCES IN DIGITAL LIBRARIES CONFERENCE, 1999, Baltimore. Proceedings... Baltimore, : [s.n.], 1999. p.19-21.

CHRISTEL, M., PENDYALA, K., Informedia goes to school : early findings from the digital video library project. D-Lib Magazine, Sept. 1996.

CHRISTEL, M., WINKLER, D., TAYLOR, R., Improving access to a digital video library. In: HUMAN COMPUTER INTERACTION, INTERACT 97 THE IFIP CONFERENCE, 1997, Sydney. Proceedings... Sydney : [s.n.], 1997.

\footnotetext{
${ }^{4} \mathrm{~A}$ maioria dos textos usados nesta revisão da literatura estão disponibilizados na URL: http://www.informedia.cs.cmu.edu/html/enter.html em formato PDF.
}

Multimedia abstrac-tions for a digital video library. In: ACM DIGITAL LIBRARIES'97 CONFERENCE, 1997, Philadelphia. Proceedings... Philadelphia : [s.n.], 1997.

DING, Wei. A study on video browsing strategies. [online]. 1997. Disponível na URL: http://www.learn.umd.edu/wp/speedex p.html

FAGUNDES, M.L.F. Videoteca multimeios : em direção a um banco de dados. Campinas,1999. 81p. Dissertação (Mestrado) - Instituto de Artes, UNICAMP.

LIENHART, R. et al. Video abstracting. Comum. ACM. v.40, n.12, p.54-62, Dec. 1997.

HAUPTMANN, A., WITBROCK, M. Story segmentation and detection of commercials in broadcast news video. In: ADL-98 ADVANCES IN DIGITAL LIBRARIES CONFERENCE, 1998, Santa Barbara, CA. Proceedings... Santa Barbara, CA : [s.n.], 1998. p.122-24.

HOUGHTON, R. Named faces : putting names to faces. IEEE Intelligent Systems Magazine, v.14, n.5, p.4550, Sept./Oct. 1999.

KOBLA, V. et al. Compressed domain video segmentation. In: KOMLODI, A,. SLAUGHER, L. Visual video browsing interfaces using key frames. Los Angeles, CA : [s.n.], Abril 1996. p.337-338. (Technical Report CARTR-839 CS-TR-3688 - University of Maryland. (Summary). 
KOMLODI, A, MARCHIONINI, G. Key frame preview techniques for video browsing. [online]. 1998. Disponível na URL: $<$ http://www.gue.umd.edu/ komlodi/d 198/d98_1.html.

O’CONNOR, B.C. Access to moving image documents : background concepts and proposals for surrogates for films. Journal of Documentation, v.41, n.4, p.209-220, 1985.

O'CONNOR, B.C. Moving image-based serial publications. Serials Review, summer \& fall, p.19-24, 1986a.

O'CONNOR, B.C. Representation and the utility of moving image documents. Proceedings of $\mathbf{4 9}^{\text {th }}$ American Society of Information Science, v.23, p.237243, 1986b

ROWLEY, H., BALUJA, S., KANADE, T. Human face detection in visual scenes. [s.1.] : Carnegie Mellon University, 1995. (Computer Science Technical Report CMU-CS-95-158).

TEODOSIO, L., BENDER, W. Salient video stills : content and context preserved. ACM Multimidea, n.6, p.39-46, 1993.

WACTLAR, H. D. New directions in video information extraction and summarization. In: DELOS Workshop, 10., 1999, Santorini. Proceedings... Santorini : [s.n], 1999. p24-25.

WITBROCK, M., HAUPTMANN, A. Improving acoustic modes by watching television. [s.1.] : Carnegie Mellon University, 1998. (Technical Report CMU CS CMU-CS-98-110).
- Speech recognition for a digital video library. Journal of the American Society for Information Science, v.49, n.7, 1998a. 